\title{
MÉCANISMES RÉGULATEURS DES $\alpha$-GLUCOSIDASES ÉPIDIDYMAIRES CHEZ LE RAT
}

\author{
Julie Morisset, Gilles Frenette et Roland R. Tremblay \\ Laboratoire de Biorégulation hormonale, Centre Hospitalier de l'Université Laval, 2705, boul. Laurier, \\ Ste-Foy, Québec, Canada, G1 V 4G2
}

REGULATORY MECHANISMS of $\alpha$-GLUCOSIDASES IN RAT EPIDIDYMIS. Epithelial cells of mammalian epididymis produced acid and neutral $\alpha$ glucosidases $(\alpha-G)$ which are either modulated by hormonal systemic influences or by local factors arising from the rete testis fluid. In this study, ligation of ductuli efferentes in the rat was characterized by a modest elevation of both $\alpha-G 15$ days after surgery. In adrenalectomized rats, both $\alpha-\mathrm{G}$ are lower than in intact animals. Because of the continuance of spermatophagia 2 weeks after ductuli ligation, the observed enzymatic variations cannot be ascribed to paracrine influences ; however, the effects of adrenalectomy on $\alpha$-G levels are obvious and may be attributed to a multifactorial endocrine control (androgens and corticoids). Key words : $\alpha$ glucosidase, epididymis, rat, hormones. Andrologie, 1991, 1 : 53-55.

Les $\alpha$-glucosidases $(\alpha-1,4$-glucosidase ; EC 3.2.1.20) épididymaires sont présentes dans le cytoplasme ou les lysosomes des cellules épithéliales bordantes des différents segments de l'organe. L' $\alpha$-glucosidase $(\alpha-G)$ neutre ou sécrétée par l'épididyme augmente chez l'homme (13) et le rat (6) en relation avec la testostérone sérique, tandis que l' $\alpha-G$ acide ou lysosomale s'abaisse en situation de photopériodisme avec testostérone élevée chez le bélier (14). Dans un travail récent $(10)$, nous avons montré que l' $\alpha-G$ acide s'élève dans les différents segments de l'épididyme du rat après une vasectomie, alors que l' $\alpha$-G demeure inchangée. Dans la présente étude, nous avons tenté de vérifier si la présence du fluide du rete testis, riche en protéines (11 $\mathrm{mg} /$ heure/testicule) (8) et en spermatozoïdes, pouvait influencer le contenu en $\alpha-G$ neutre de la tête ou de la queue de l'épididyme par un mécanisme relevant de la communication paracrine. Plusieurs protéines spécifiques à ce fluide sont en effet réabsorbées au niveau de l'épithélium de la tête de l'épididyme (12) et pourraient ainsi influencer l'activité de sécrétion des différentes cellules bordantes.

\section{MATERIELS ET METHODES}

Des rats de souche Sprague-Dawley dont le poids initial a varié entre 200 et $225 \mathrm{~g}$ ont été utilisés dans ce travail. Ils étaient gardés en cage individuelle, soumis à un cycle d'éclairage : obscurité de $14: 12$ heures, maintenus dans des conditions de température et d'humidité constantes, nourris et abreuvés ad libitum. Les interventions chirurgicales furent toutes pratiquées sous anesthésie au pentobarbital à raison de $0.04 \mathrm{mg} / \mathrm{g}$ de poids corporel et ont comporté les actes suivants :

1. Vasectomie bilatérale. Elle fut pratiquée selon la méthode de Flickinger et coll. (1972).

2. Ligature des ductuli efferentes. Le pédicule reliant le testitule et la tête de l'épididyme fut soigueusement disséqué avec de microinstruments de chirurgie et sous amplification (x10) avec des loupes à prisme $\mathrm{E}$ de la compagnie Zeiss. Après identification des structures vasculaires et des canalicules, ces derniers furent ligaturés avec une soie 5-0. Le testicule bien vascularisé fut remis en position intrascrotale. Cette ligature fut effectuée chez deux groupes d'animaux : intacts ou surrénalectomisés par voie lombaire.

Les animaux furent sacrifiés par décapitation (guillotine Harvard) 15 jours après le début des manipulations. Les testicules et les épididymes furent prélevés rapidement, déposés sur glace et pesés. L'épididyme fut divisé immédiatement en deux segments, tête et queue, et conservé à $-80^{\circ} \mathrm{C}$ jusqu'au dosage de l'activité $\alpha$-glucosidase. Dans chacun des groupes d'animaux, deux épididymes furent fixés au formol pour coloration à l'hématoxyline-éosine ou dans la glutaraldéhyde $5 \%$ et la formaldéhyde $4 \%$ dans un tampon cacodylate $1 \mathrm{M}, \mathrm{pH} 7.3$, pendant 1 heure, pour observation en microscopie électronique.

L'activité $\alpha$-glucosidase fut déterminée à $\mathrm{pH}$ optimum dans le milieu d'incubation, tel que décrit par Chapdelaine et coll. (3) ; cette technique fut modifiée par Grandmont et coll. (6) pour les mesures d'activité enzymatique dans le cytosol des tissus de l'appareil reproducteur du rat. Le contenu protéique du cytosol fut évalué par la méthode de Lowry et coll. (9) en utilisant l'albumine sérique bovine comme référence. Le degré de signification statistique des résultats fut obtenu par analyse de variance précédée d'un test d'homogénéité de la variance.

\section{RESULTATS}

1. Effet de la vasectomie et des interventions chirurgicales sur le poids de l'épididyme.

Chez les animaux vasectomisés, le poids des épididymes droit et gauche fut rigoureusement comparable à celui des animaux contrôles (Table 1). Par contre une diminution de l'ordre de $25 \%$ du poids de l'organe fut observée après ligature des ductuli efferentes chez les rats intacts ou surrénalectomisés. La castration entraîne une perte de poids de l'épididyme de $60 \%$ par référence aux animaux intacts du même âge .

Tableau 1 : Poids des épididymes (E) lors du sacrifice des animaux*

\begin{tabular}{lcc}
\hline & $\begin{array}{c}\text { E droit** } \\
(\mathrm{mg})\end{array}$ & $\begin{array}{c}\text { E gauche** } \\
(\mathrm{mg})\end{array}$ \\
\hline Intacts & $0.38 \pm 0.09$ & $0.37 \pm 0.08$ \\
Vasectomie & $0.35 \pm 0.04$ & $0.34 \pm 0.03$ \\
Castration & $0.14 \pm 0,01$ & $0.15 \pm 0.01$ \\
Ligature ductuli & $0.29 \pm 0.08$ & $0.26 \pm 0.05$ \\
efferentes (DE) & & \\
Ligature des DE + & $0.29 \pm 0.07$ & $0.28 \pm 0.03$ \\
Surrénalectomie & \\
* Le nombre d'observations a varié entre 10 et 12 par groupe \\
** Moyenne \pm erreur-type de la moyenne. \\
\hline
\end{tabular}

\section{Influence des interventions chirurgicales} sur l' $\alpha$ - $G$ acide de l'épididyme.

Tel que rapporté récemment par Marchand et coll. (10), l' $\alpha$-G acide s'élève tant au niveau de la tête que de la queue de l'épididyme après vasectomie. La ligature des ductuli efferentes (DE) est associée à une élévation significative de $l^{\prime} \alpha$-G acide au niveau de la tête (Fig. 1, section A) et non de la queue (Fig. 1, section B) de l'organe. La surrénalectomie bilatérale entraîne une baisse significative de l' $\alpha-G$ dans les deux segments de l'organe (DE + ADR-X), tel qu'illustré sur la figure 1 .

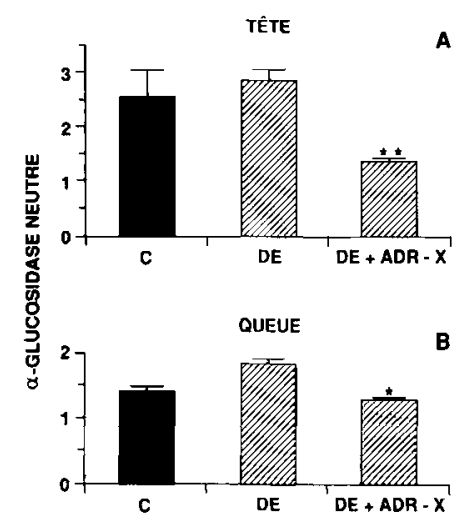

Figure 1: Effet de la ligature des ductuli efferentes (DE) et de la surrénalectomie bilatérale (DE + ADR-X) sur l'activité $\alpha$-1,4-glucosidase acide de la tête (section A) et de la queue (section B) de l'épididyme. 
3. Influence des interventions chirurgicales sur I' $\alpha$-G neutre de l'épididyme.

La vasectomie n'a aucun effet sur le contenu en $\alpha-G$ neutre de l'épididyme tant dans cette étude que dans le travail de Marchand et coll. (10). Toutefois, la ligature des ductuli efferentes s'accompagne d'une augmentation de l' $\alpha-\mathrm{G}$ neutre de 23 et $27 \%$ respectivement pour la tête et la queue de l'épididyme par référence aux valeurs des animaux intacts. La surrénalectomie bilatérale annule complètement cet effet (Fig. 2, section $\mathrm{A}$ et $\mathrm{B}$ ).

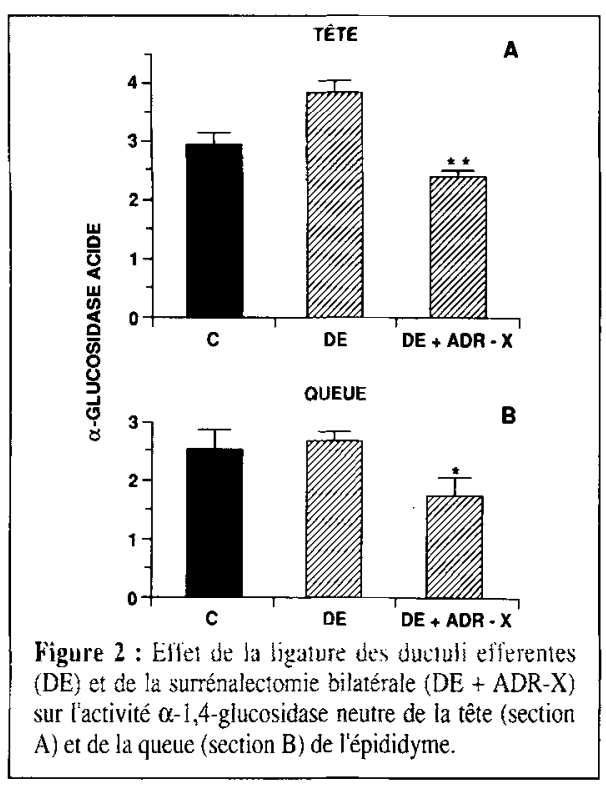

\section{Aspects morphologiques}

Après ligature des ductuli efferentes, la lumière du tubule épididymaire est réduite et présente de nombreux replis de son épithélium. L'aspect de ces cellules apparaît comparable à celui des animaux intacts. Aucun spermatozoïde n'est identifiable dans tout l'organe.

Au plan ultrastructural, la vasectomie entrâne une forte accumulation de corps denses intracytoplasmiques compatibles avec des lysosomes qui sont plus proéminents au niveau de la tête que de la queue de l'épididyme. La taille des lysosomes est d'emblée supérieure à celle des mêmes organelles chez des animaux intacts. Après la ligature des ductuli efferentes, tant les cellules principales que les cellules claires de l'épididyme sont le siège de nombreuses vacuoles pâles et remplies de matériel hétérogène. Le système mitochondrial et l'appareil de Golgi de ces cellules sont très développés par opposition aux observations réalisées dans les cellules intactes. Les corps denses de type lysosome ne sont pas modifiés dans tout l'épithélium épididymaire à la suite de la ligature des ductuli efferentes mais il y a persistance de spermatophagie dans l'organe (résultats non montrés).

\section{DISCUSSION}

La problématique centrale de ce travail étant reliée à l'influence de la ligature des ductuli efferentes sur les activités $\alpha$-G neutre et acide du tissu épididymaire ne doit pas nous éloigner de certaines constatations accessoires suscitées par la simple vasectomie. Cette dernière procédure chirurgicale entraîne une augmentation sélective de l' $\alpha-G$ acide de l'épididyme et nous rapportons pour la première fois que l'absence de corticostérone circulante prévient cet effet. L'élévation de l' $\alpha-G$ acide paraissait bien refléter une activité lysosomale accrue, selon nos observations avec la mesure de la cathepsine D (10), ces faits étant indirectement corroborés par nos analyses ultra-structurales et celles de plusieurs auteurs $(1,5,7)$ associant spermatophagie et modifications de l'appareil lysosomal. Or, la ligature des ductuli efferentes entraîne également une augmentation de $l^{\prime} \alpha-G$ acide épididymaire et il est possible de faire intervenir le même déterminant puisque la spermatophagie perdure encore dans la queue de l'épididyme. D'où la difficulté d'impliquer un mécanisme de contrôle paracrinien pour l' $\alpha-\mathrm{G}$ acide dans la fenêtre d'observation sélectionnée. Seule l'absence de corticostérone permet de suggérer une influence endocrine sur le mode d'expression ou le turnover du messager de l'enzyme.

La ligature des ductuli efferentes et la vasectomie ont-elles des effets analogues sur la modulation de l' $\alpha$-G neutre ? Il nous est connu que la vasectomie n'influence pas la forme sécrétoire de l'enzyme, alors que l'exclusion du fluide du rete testis, qui prive l'épididyme de son apport en spermatozoïdes, protéines (8) et hormones androgéniques $(2,16)$, s'accompagne d'une tendance à l'élévation de l' $\alpha$-G neutre tant au niveau de la tête que de la queue de l'épididyme. Cette tendance est à nouveau contrecarrée par l'absence de corticostérone endogène. Dans ce type d'expérience, la castration entraîne évidemment des abaissements d' $\alpha-G$ neutre beaucoup plus drastiques que la surrénalectomie. Cette observation permet de souligner une importante asymétrie dans la réponse de l'épithélium épididymaire aux stéroïdes, l'influence dominante devant être attribuée aux androgènes circulants et non à ceux libérés dans le rete testis ; par ailleurs, l'effet plutôt modeste de l'absence de fluide du rete testis sur l' $\alpha-G$ neutre tissulaire demeure difficile à interpréter en termes de contrôle paracrinien sans le support de l'immunohistochimie et de la biologie moléculaire pour évaluer le contenu épididymaire en ARN messager spécifique pour l'a-G. A prime abord, toute intervention chirurgicale au niveau du rete testis pourrait s'accompagner d'une réaction inflammatoire diffuse de l'organe et entrainer une baisse de l' $\alpha-G$ sécrétée, tel que rapporté pour l'épididyme humain (4). Dans notre étude, aucune infiltration leuco-lymphocytaire n'a été observée deux semaines après la ligature des ductuli efferentes. Il faut donc présumer avec Vanha-Perttula (15) que la fonction sécrétoire des cellules principales de l'épididyme a été maintenue, sinon augmentée $(25 \%)$ pour l' $\alpha$-G neutre. Dans l'état actuel de nos connaissances, cette observation s'inscrit à l'opposé du contrôle paracrinien exercé par les cellules de Sertoli et/ou les cellules germinales primaires sur la $5 \alpha$-réductase nucléaire de l'épididyme du rat (11).

En résumé, les résultats de la présente étude indiquent qu'en sus de l'influence des androgènes sur la fonction épididymaire, il y a intérêt à prendre en compte le rôle des corticoïdes sur la modulation de la sécrétion des enzymes solubles ou d'origine lyso-somale de cet épithélium. Ces facteurs systémiques pourraient être plus facilement décelables que les déterminants véhiculés dans le fluide du rete testis.

\section{REMERCIEMENTS}

Nous tenons à exprimer notre gratitude à Madame Lucie Turcotte pour la préparation de ce manuscrit.

Recherche subventionnée par le Fonds de la Recherche en Santé du Québec, Canada.

\section{REFERENCES}

1 - Alexander NJ. Ultrastructural changes in rat epididymis after vasectomy. Z. Zellforsched, 1973, 136: 177-182.

2 - Back DJ. The presence of metabolites of $3 \mathrm{H}$ testosterone in the lumen of cauda epididymis of the rat. Steroids, 1975, 25:413-420.

3- Chapdelaine P, Tremblay RR, Dubé JY. Origine of maltase and variations in infertile men. Arch. Androl., 1978, 1:61-68.

4- Cooper TG, Weidner W, Nieschlag AG. The influence of inflammation on the human male genital tract on secretion of the seminal markers $\alpha$-glucosidase, glycerophosphocholine, carnitine, fructose and citric acid. Int. J. Andrology, 1990, $13: 329-336$.

5- Flickinger CJ, Gallien TN, Spell DR. The incidence of spermatic granulomas and their relation to testis weight after vasectomy and vasovasostomy in Lewis rats. J. Androl, 1986, $7: 285-291$.

6- Grandmont AM, ChapdeJaine P, Tremblay $R R$. Presence of $\alpha$-glucosidases in the male reproductive system of the rat and hormonal influences. Can. J. Biochem., 1983, 61:764-769.

7- Hoffer AP, Hamilton DW, Fawcett DW Phagocytosis of spermatozoa by the epithelial cells of the ductuli efferentes after epididymal obstruction in the rat. J. Reprod. Fertil., 1975. $44: 1-9$. 
8 - Koskimies AI, Kormano M. The proteins in fluids from the semineferous tubules and rete testis of the rat. J. Reprod. Fertil., 1973, 34 : 433-444.

9 - Lowry OH, Rosebrough NJ, Farr AL, Randall RJ. Protein measurement with the Folin phenol reagent. J. Biol. Chem., 1951, 193 : 265-275.

10 -Marchand L., Frenette G, Tremblay RR. Fate of $\alpha-1,4$ glucosidase and cathepsin $D$ in the rat epididymis after vasectomy. Enzyme, 1990, 43:192-196.

11 -Robaire B., Hermo L. Efferent ducts, epididymis, and vas deferens : structure, functions, and their regulation. The Physiology of Reproduction, Knobil E et Neil J eds, Raven Press, 1988. Chap. 23: 999-1081.

12 -Shabanowitz RB, Killian GJ. Two dimensional electrophoresis of proteins in principal cells, spermatozoa, and fluid associated with the rat epididymis. Biol. of Reprod., 1987, 36: 753-768.

13 -Tremblay RR. Epididymal markers and sperm analysis. In Andrology, Male Fertility and Sterility. Eds JD Paulson, A Negro-Vilar, E Lucena et L Martini, 1986 : 273-292.
14 -Tremblay RR, Demers P, Besançon J, Lemay JP. Alpha-1,4-glucosidase activity in ram seminal plasma is inversely related to serum testosterone. Enzyme, 1990, 43 (2) : 107-111.

15 -Vanha-Perttula T, Arya M. Lectin staining of rat testis and epididymis after ligation of excurrent ducts at different levels. Biol. Reprod., 1985, $33:$ 477-485.

16-Vreeburg JTM. Distribution of testosterone and $5 \alpha$-dihydrotestosterone in rat epididymis and their concentrations in efferent duct fluid. J. Endocrinol., 1975, 67 : 203-210.
RESUME : Les cellules épithéliales de l'épididyme des mammifères contiennent des $\alpha$-glucosidases $(\alpha-G)$ acide et neutre dont la modulation obéit à un contrôle hormonal systémique ou encore local par le biais du fluide du rete testis. Dans ce travail, la ligature des ductuli efférentes chez le rat est caractérisée par une élévation modeste des deux $\alpha-G 15$ jours après la chirurgie. Chez des animaux surrénalectomisés, les activités $\alpha-G$ s'abaissent par référence aux valeurs retrouvées chez l'animal intact. Etant donné la persistance de spermatophagie deux semaines après ligature des ductuli, les variations enzymatiques observées sont difficilement attribuables à des influences paracrines ; par contre, les effets de l'absence de corticostérone sur les $\alpha-\mathrm{G}$ sont tangibles et reflètent un contrôle stéroïdien multifactoriel (androgènes et corticoïdes). Mots clefs : $\alpha$-glucosidase, épididyme, rat, hormones. Andrologie, 1991, 1 : 53-55.

\section{INSTRUCTIONS AUX AUTEURS}

Les textes scientifiques publiés dans Andrologie doivent être constitués comme suit, et adressés en deux exemplaires au rédacteur en chef :

1. Page de garde comportant titre, nom(s), prénom(s) et adresse(s) de(s) institution(s) de(s) auteur(s).

2. Page suivante comportant un titre anglais, un résumé anglais plus long et détaillé que le résumé français, mais ne devant pas dépasser 30) lignes dactylographiées double interligne, et une liste de 2 à 6 mots clés en anglais.

3. A partir de la page suivante, texte dactylographié en double interligne sur un seul côté de la page, et selon un format normalisé : 27 lignes de 60 caractères et espaces par ligne. Chaque fois que possible, la présentation doit suivre un plan classique, c'est à dire : Introduction, Matériel et méthodes, Résultats, Discussion, Références. Le texte doit être le plus concis possible.

4. Les références sont à présenter sous le titre "REFERENCES", et par ordre alphabétique. Elles soivent être appelées dans le texte par leur numéro. Leur nombre doit être limité à 10 au maximum pour 4 pages de texte ( 20 pour 8 pages, 30 pour 12 pages, sauf cas particulier de revue générale). Suivre le modèle ci-après :

1/ Auroux M, Dulioust E. Cyclophosphamide in the male rat : Behavioral effects in the adults in artificial insemination. In : David G, Price WS eds. Human artificial insemination and semen preservation. New York, Plenum Press, 1980: 197-210.

2/ Schwartz D, Mayaux MJ. Mode of evaluation of results in artificial insemination. In : David G, Price WS eds. Human artificial insemination and semen preservation. New York, Plenum Press, 1980 : 197-210.

5. Sur la page suivant les références, placer un résumé en français court, maximum 10 lignes dactylographiées double interligne, suivi d'une liste de 2 à 6 mots clés en français.

6. Les tableaux et figures doivent être présentés à part, 1 par page. Les légendes des différentes figures doivent être dactylographiées sur une page séparée. Le numéro des figures doit être inscrit au dos de chacune, au crayon de bois. Les photos en noir et blanc peuvent être reproduites, mais pas les photos et illustrations. 